\title{
Mechanical and thermal properties of environmentally friendly composites derived from sugar palm tree.
}

\begin{abstract}
The aim of this paper is to study the effect of fibre content on mechanical properties, water absorption behaviour and thermal properties of sugar palm fibre (SPF) reinforced plasticized sugar palm starch (SPF/SPS) biocomposites. The biocomposites were prepared with different amounts of fibres (i.e. 10\%, $20 \%$ and $30 \%$ by weight percent) by using glycerol as plasticizer for the starch. The mechanical properties of plasticized SPS improved with the incorporation of fibres. Fibre loading also increased the thermal stability of the biocomposite in this investigation. Water uptake and moisture content of SPF/SPS biocomposites decreased with the incorporation of fibres, which is due to better interfacial bonding between the matrix and fibres as well as the hindrance to absorption caused by the fibres. Fractographic studies through scanning electron microscopy showed homogeneous distribution of fibres and matrix with good adhesion which play an important role in improving the mechanical properties of biocomposites.
\end{abstract}

Keyword: Mechanical; Thermal; Absorption; Adhesion; Biocomposites 